\title{
Spatially Resolved Magnetic Field Structure in the Disk of a T Tauri Star
}

Ian W. Stephens ${ }^{1}$, , Leslie W. Looney ${ }^{2}$, Woojin Kwon ${ }^{3}$, Manuel Fernández-López ${ }^{2}$, A. Meredith Hughes $^{5}$, Lee G. Mundy ${ }^{6}$, Richard M. Crutcher ${ }^{2}$, Zhi-Yun Li $^{7}, \&$ Ramprasad Rao ${ }^{8}$

${ }^{1}$ Institute for Astrophysical Research, Boston University, Boston, MA 02215, USA

${ }^{2}$ Department of Astronomy, University of Illinois, Urbana, Illinois 61801, USA

${ }^{3}$ SRON Netherlands Institute for Space Research, Landleven 12, 9747 AD Groningen, The Netherlands

${ }^{4}$ Instituto Argentino de Radioastronomía, CCT-La Plata (CONICET), C.C.5, 1894, Villa Elisa, Argentina

${ }^{5}$ Van Vleck Observatory, Astronomy Department, Wesleyan University, Middletown, CT 06459, USA

${ }^{6}$ Astronomy Department \& Laboratory for Millimeter-wave Astronomy, University of Maryland, College Park, MD 20742, USA

${ }^{7}$ Astronomy Department, University of Virginia, Charlottesville, VA 22904, USA

${ }^{8}$ Institute of Astronomy and Astrophysics, Academia Sinica, Hilo, HI 96720, USA

Accepted for publication in Nature

Magnetic fields in accretion disks play a dominant role during the star formation process ${ }^{12}$ but have hitherto been observationally poorly constrained. Field strengths have been inferred on $T$ Tauri stars themselves ${ }^{3}$ and possibly in the innermost part of the accretion disk $^{\sqrt{4}}$, but 
the strength and morphology of the field in the bulk of the disk have not been observed. Unresolved measurements of polarized emission (arising from elongated dust grains aligned perpendicular to the field ${ }^{5}$ ) imply average fields aligned with the disks ${ }^{67}$. Theoretically, the fields are expected to be largely toroidal, poloidal, or a mixture of the two ${ }^{1 / 28}+10$, which imply different mechanisms for transporting angular momentum in the disks of actively accreting young stars such as HL Tau ${ }^{11}$. Here we report resolved measurements of the polarized 1.25 mm continuum emission from HL Tau's disk. The magnetic field on a scale of $80 \mathrm{AU}$ is coincident with the major axis $\left(\sim 210 \mathrm{AU}\right.$ diameter $\left.{ }^{12}\right)$ of the disk. From this we conclude that the magnetic field inside the disk at this scale cannot be dominated by a vertical component, though a purely toroidal field does not fit the data well either. The unexpected morphology suggests that the magnetic field's role for the accretion of a T Tauri star is more complex than the current theoretical understanding.

HL Tau is located 140 pc away ${ }^{13}$ in the Taurus molecular cloud. Although HL Tau is a $\mathrm{T}$ Tauri star, it is considered to be an early example due to its bipolar outflow ${ }^{14}$ and possible residual envelope $\mathrm{e}^{15}$. Observations and modeling of the protostar with a thick, flared disk suggest a stellar mass of $\sim 0.55 M_{\odot}$ and a disk mass of $0.14 M_{\odot}^{[12}$. A possible planet forming in HL Tau's disk has been observed ${ }^{16}$, though this detection was not confirmed ${ }^{12}$. However, HL Tau's disk is gravitationally unstable which could favor fragmentation into Jupiter mass planets ${ }^{12,16}$. HL Tau has the brightest $\mathrm{T}$ Tauri star disk at millimeter wavelengths, allowing for the best possible probe of the fractional polarization $P$. Previous observations of the polarization of HL Tau's disk yielded marginally significant, spatially unresolved polarization detections with the James Clerk Maxwell 
Telescope (JCMT, $P=3.6 \% \pm 2.4 \%$ at $14^{\prime \prime}=1960$ AU resolution) $)^{\sqrt[6]{6}}$ and the Submillimeter Array $\left(\mathrm{SMA}, P=0.86 \% \pm 0.4 \%\right.$ at $2^{\prime \prime}=280 \mathrm{AU}$ resolution, archival observations released in this paper). In addition, HL Tau observations with the Combined Array for Millimeter-wave Astronomy (CARMA) have shown that the interferometric emission comes entirely from the disk with no contamination of large-scale envelope emission ${ }^{12}$. HL Tau is therefore a very promising source to search for a resolved polarization detection.

Only through observations of polarized dust emission can the morphology of the magnetic field be ascertained, though higher resolution dust polarimetric observations of T Tauri star disks do not detect polarization and place stringent upper limits $(P<1 \%)$ on the polarization fraction ${ }^{17}, 18$, which disagree with theoretical models of high efficiency grain alignment with a purely toroidal field $^{8}$. There is a clear discrepancy between theoretical models of the magnetic fields in disks and the observations to-date, requiring more sensitive observations of the dust polarization. The SMA recently detected the magnetic field morphology in the circumstellar disk of the Class 0 (i.e., the earliest protostellar stage) protostar IRAS $16293-2422 \mathrm{~B}^{19}$, but since this disk is nearly face-on, observations cannot detect the vertical component of the magnetic field. Moreover, this source is perhaps one of the youngest of the known Class 0 sources $\frac{20}{}$, increasing the chances that the polarized flux could be from the natal environment. Nevertheless, since the disk is the brightest component at the probed scale, polarization most likely comes from the disk, and the magnetic field morphology hints at toroidal wrapping 19 .

We have obtained 1.25 mm CARMA polarimetric maps of HL Tau at $0.6^{\prime \prime}$ (84 AU) resolution 
and plotted the magnetic field morphology in Figure 1. This is a resolved detection (with approximately 3 independent beams) of the magnetic field morphology in the circumstellar disk of a $\mathrm{T}$ Tauri star. The central magnetic field vector has a measured position angle (PA, measured counterclockwise from north) of $\theta_{B}=143.6^{\circ} \pm 4.4^{\circ}$, which is within $9^{\circ}$ of the previously measured PA of the major axis of the HL Tau disk ${ }^{12}$. This angle is in agreement with that measured by the JCMT $\left(\theta_{B}=140^{\circ} \pm 20^{\circ}\right)^{6}$ and archival observations from the SMA analyzed here $\left(\theta_{B}=137^{\circ} \pm 13^{\circ}\right)$. The central vector has a fractional polarization of $P=0.59 \% \pm 0.09 \%$, and $P$ varies over the disk between $0.54 \% \pm 0.13 \%$ and $2.4 \% \pm 0.7 \%$ with an average and median $P$ of $0.90 \%$ and $0.72 \%$ respectively, in agreement with the upper limits $(P<1 \%)$ of other T Tauri star polarimetric observations ${ }^{17118}$. This median value is significantly less than IRAS $16293-2422$ B (1.4\%) which could indicate disk evolution; the dust grains could grow larger and become more spherical with time, and/or the magnetic field is becoming more turbulent.

To constrain the intrinsic magnetic field configuration inside HL Tau's disk, we made a simple model which incorporates a combination of a toroidal and a vertical component; a radial field component inside the bulk of the disk (most likely probed by our polarization observation) is expected to be sheared quickly into a toroidal configuration by differential rotation on the short time-scale of the disk rotation. We use the best estimates of disk parameters ${ }^{12}\left(\mathrm{PA}=136^{\circ}\right.$ and inclination $i=40^{\circ}$, both accurate within a few degrees) and vary the relative strength of the two field components from $100 \%$ toroidal and $0 \%$ vertical to $0 \%$ toroidal and $100 \%$ vertical in $1 \%$ increments (modeling details are in the Methods section). Using the $\geq 3 \sigma$ detections in Figure 1, we find that a completely toroidal field is the best fit model. However, the reduced $\chi$ value is 
high (69, where a value of 1 indicates an optimal fit). If we do not constrain the magnetic field to be aligned with the best-fit $\operatorname{disk}^{12}$ we can achieve a better fit. For a disk with a PA of $151^{\circ}$, the best fit model to the observations is almost completely edge on, with an inclination of $i=89^{\circ}$ (reduced $\chi$ value of 1). Figure 2 shows the observed versus the modeled parameters for PA of $136^{\circ}$ and $i=40^{\circ}$ as well as PA of $151^{\circ}$ and $i=89^{\circ}$. While the PA does not vary largely from the major axis of the disk ( $15^{\circ}$ difference), $i=89^{\circ}$ is inconsistent with our disk dimensions and the well-constrained continuum observations of other studies ${ }^{12} 21$. There is also no detected polarized flux in the northeast (upper-left) and southwest (bottom-right) of the disk where $P$ for a toroidal field should be the highest due to less beam smearing. This fact, along with the poor fit with the constrained disk parameters, indicates that although there is probably a toroidal component, the field apparently has substantial contributions from other components.

We note that adding a vertical component to all our models makes the fit worse. The lack of any vertical component in the model suggests that dominant poloidal fields are likely absent inside the disk since, as we argued earlier on dynamical grounds, a predominantly radial field is unlikely inside the disk because of rapid differential rotation. Moreover, although points with $>5 \sigma$ polarization detections fit a toroidal morphology even for the $i=40^{\circ}$ model, we cannot be sure that the disk field is only toroidal since a straight, uniform field in the plane of the disk, although physically unlikely for a disk system, also well fits the data.

If the disk of HL Tau has a dominant toroidal component, then it is uncertain what causes the outer vectors to not fit the toroidal morphology based on HL Tau's best fit disk dust model ${ }^{12}$ 
(Figure 2, left). Perhaps the grains in some parts of the disk do not efficiently align with the disk field. Another possibility is that the magnetic field toward the inner disk is toroidal and beamaveraged to be aligned with the major axis of the disk. However, toward the edge of the disk, where the disk field may be less tightly wound and weaker, the magnetic field may be dominated by external field lines that are already toroidal (e.g., due to a rotating envelope). The incoming fields may strongly influence or even dominate the magnetic field in the outer disk. In any case, the discrepancy indicates that, at least for this particular source, one needs to go beyond the simplest magnetorotational instability ${ }^{2}$ disk models that do not include any external influence or distortion. Both theoretical studies tailored to HL Tau and higher resolution polarization observations are needed to resolve this puzzle.

At the 1000 AU scale, structured magnetic fields are observed around young, low-mass $\operatorname{protostars}^{22.23}$, but fields appear to be randomly aligned with respect to the inferred disk ${ }^{24 \sqrt[25]{ }}$. Misalignment of the field lines with the rotation axis can help overcome magnetic braking to create a centrifugally supported disk at the 100 AU scale ${ }^{10}$. Further disk evolution can be driven by magnetorotational instability ${ }^{2}$ or a magnetocentrifugal wind ${ }^{19}$. A toroidally dominant disk field is expected in the former scenario, and a significant poloidal field is required for the latter (at least near the wind launching surface). Both processes can possibly contribute to the disk accretion at the same time.

Until now, we have been unable to observationally constrain the magnetic field morphology in disks. Along with the Class 0 source IRAS 16293-2422 B, the observations of HL Tau show 
that a toroidal field component may last from the low-mass protostar's initial formation to the T Tauri star stage - approximately the first $10^{6}$ years of a protostar's life $\mathrm{e}^{26}$. The apparent absence of vertical fields for these observations implies that magnetocentrifugal winds driven along largescale poloidal magnetic fields ${ }^{27}$ are probably not the dominant mechanism for redistributing the disk's angular momentum during the accretion process of a star at the probed 80 AU size-scale. However, the morphology detected in HL Tau cannot be fully explained by a simple mix of toroidal and vertical components either, requiring future observations at both large scale and small scale to truly understand the role of magnetic fields in the formation of solar systems like our own.

1. Blandford, R. D. \& Payne, D. G. Hydromagnetic flows from accretion discs and the production of radio jets. Mon. Not. R. Astron. Soc. 199, 883-903 (1982).

2. Balbus, S. A. \& Hawley, J. F. Instability, turbulence, and enhanced transport in accretion disks. Reviews of Modern Physics 70, 1-53 (1998).

3. Johns-Krull, C. M. The Magnetic Fields of Classical T Tauri Stars. Astrophys. J. 664,975-985 (2007).

4. Donati, J.-F., Paletou, F., Bouvier, J. \& Ferreira, J. Direct detection of a magnetic field in the innermost regions of an accretion disk. Nature 438, 466-469 (2005).

5. Lazarian, A. Tracing magnetic fields with aligned grains. Journal of Quantitative Spectroscopy \& Radiative Transfer 106, 225-256 (2007). 
6. Tamura, M., Hough, J. H. \& Hayashi, S. S. 1 Millimeter Polarimetry of Young Stellar Objects: Low-Mass Protostars and T Tauri Stars. Astrophys. J. 448, 346 (1995).

7. Tamura, M. et al. First Detection of Submillimeter Polarization from T Tauri Stars. Astrophys. $J .525,832-836$ (1999).

8. Cho, J. \& Lazarian, A. Grain Alignment and Polarized Emission from Magnetized T Tauri Disks. Astrophys. J. 669, 1085-1097 (2007).

9. Konigl, A. \& Pudritz, R. E. Disk Winds and the Accretion-Outflow Connection. Protostars and Planets IV 759 (2000).

10. Hennebelle, P. \& Ciardi, A. Disk formation during collapse of magnetized protostellar cores. Astron. Astrophys. 506, L29-L32 (2009).

11. Robitaille, T. P., Whitney, B. A., Indebetouw, R. \& Wood, K. Interpreting Spectral Energy Distributions from Young Stellar Objects. II. Fitting Observed SEDs Using a Large Grid of Precomputed Models. Astrophys. J. Supplement 169, 328-352 (2007).

12. Kwon, W., Looney, L. W. \& Mundy, L. G. Resolving the Circumstellar Disk of HL Tauri at Millimeter Wavelengths. Astrophys. J. 741, 3 (2011).

13. Rebull, L. M., Wolff, S. C. \& Strom, S. E. Stellar Rotation in Young Clusters: The First 4 Million Years. Astron J. 127, 1029-1051 (2004).

14. Movsessian, T. A., Magakian, T. Y. \& Moiseev, A. V. Kinematics and the origin of the internal structures in HL Tauri jet (HH 151). Astron. Astrophys. 541, A16 (2012). 
15. Welch, W. J., Hartmann, L., Helfer, T. \& Briceño, C. High-Resolution, Wide-Field Imaging of the HL Tauri Environment in ${ }^{13} \mathrm{CO}(1-0)$. Astrophys. J. 540, 362-371 (2000).

16. Greaves, J. S., Richards, A. M. S., Rice, W. K. M. \& Muxlow, T. W. B. Enhanced dust emission in the HL Tau disc: a low-mass companion in formation? Mon. Not. R. Astron. Soc. 391, L74-L78 (2008).

17. Hughes, A. M. et al. A Spatially Resolved Inner Hole in the Disk Around GM Aurigae. Astrophys. J. 698, 131-142 (2009).

18. Hughes, A. M., Hull, C. L. H., Wilner, D. J. \& Plambeck, R. L. Interferometric Upper Limits on Millimeter Polarization of the Disks around DG Tau, GM Aur, and MWC 480. Astronom. $J .145,115$ (2013).

19. Rao, R., Girart, J. M., Lai, S.-P. \& Marrone, D. P. Detection of a Magnetized Disk around a Very Young Protostar. Astrophys. J. L. 780, L6 (2014).

20. Loinard, L. et al. ALMA and VLA observations of the outflows in IRAS 16293-2422. Mon. Not. R. Astron. Soc. 430, L10-L14 (2013).

21. Lay, O. P., Carlstrom, J. E. \& Hills, R. E. Constraints on the HL Tauri Protostellar Disk from Millimeter- and Submillimeter-Wave Interferometry. Astrophys. J. 489, 917 (1997).

22. Girart, J. M., Rao, R. \& Marrone, D. P. Magnetic Fields in the Formation of Sun-Like Stars. Science 313, 812-814 (2006). 
23. Stephens, I. W. et al. The Magnetic Field Morphology of the Class 0 Protostar L1157-mm. Astrophys. J. L. 769, L15 (2013).

24. Hull, C. L. H. et al. Misalignment of Magnetic Fields and Outflows in Protostellar Cores. Astrophys. J. 768, 159 (2013).

25. Hull, C. L. H. et al. TADPOL: A $1.3 \mathrm{~mm}$ Survey of Dust Polarization in Star-forming Cores and Regions. Astrophys. J. Supplement 213, 13 (2014).

26. Evans, N. J., II et al. The Spitzer c2d Legacy Results: Star-Formation Rates and Efficiencies; Evolution and Lifetimes. Astrophys. J. Supplement 181, 321-350 (2009).

27. Wardle, M. \& Koenigl, A. The structure of protostellar accretion disks and the origin of bipolar flows. Astrophys. J. 410, 218-238 (1993).

Supplementary Information is available in the online version of the paper.

Acknowledgements We acknowledge Richard L. Plambeck and Charles L. H. Hull for their consultation during the data reduction process and Charles F. Gammie for helpful discussions. This research made use of APLpy, an open-source plotting package for Python hosted at http://aplpy.github.com. Illinois and Maryland were supported by NSF AST-1139950 and AST-1139998 respectively. Support for CARMA construction was derived from the states of California, Illinois, and Maryland, the James S. McDonnell Foundation, the Gordon and Betty Moore Foundation, the Kenneth T. and Eileen L. Norris Foundation, the University of Chicago, the Associates of the California Institute of Technology, and the National Science Foundation. Ongoing CARMA development and operations are supported by the National Science Foundation under a cooperative agreement (NSF AST 08-38226) and by the CARMA partner universities. 
Author Contributions Data acquisition and reduction was performed by I. Stephens, L. Looney, and M. Fernández-López. Polarization modeling was performed by W. Kwon and fitted by I. Stephens. All authors analyzed and discussed the observations and manuscript.

Author Information Reprints and permissions information is available at www.nature.com/reprints. The authors declare no competing financial interests. Readers are welcome to comment on the online version of the paper. Correspondence and requests for materials should be addressed to I. Stephens. (ianws@bu.edu). 


\section{Methods}

Data Reduction. The CARMA dual polarization receivers allow for the measurement of polarized dust emission. Polarimetric maps can thus be created, which provide the flux density (Stokes $I$ ), the PA of the dust polarization, and $P$ at every point within the map. CARMA continuum observations in Full Stokes mode were taken at $237 \mathrm{GHz}$ in two different resolutions with each resolution having multiple tracks. The C-array observations $\left(\sim 0.79^{\prime \prime}\right.$ resolution) consisted of four tracks in October and November 2013 while the B-array observations ( $\sim 0.37^{\prime \prime}$ resolution) consisted of three more tracks in November and December 2013.

We have reduced the CARMA observations using the MIRIAD package ${ }^{28}$. The dual-polarization receivers of CARMA measure right- $(\mathrm{R})$ and left-circular (L) polarization and the four crosspolarization (RR, LL, LR, RL) terms. In order to calibrate CARMA Full Stokes observations, apart from the usual interferometric calibrations (bandpass, phase, and flux corrections), two additional calibrations are required: XYPhase (due to $\mathrm{L}$ and $\mathrm{R}$ channel phase differences) and leakage (due to $\mathrm{L}$ and $\mathrm{R}$ channels cross-coupling). These calibrations were done in the typical manner for CARMA ${ }^{25}$. The leakage terms for each antenna were consistent from track to track, and the overall accuracy of the leakage calibrations are expected to be approximately $0.1 \%$. Day to day consistency in polarization observations was tested by measuring the phase calibrator, $0510+180$. The polarization angle changed slightly from day to day, with a steady increase of a few degrees with each newer track. Intraday variability is a well-known phenomenon which affects the total flux density, the linearly polarized flux density, and the polarization angle ${ }^{29}$ and likely explains the variations of a few degrees seen in $0510+180$ from track to track. Since the variation of the polar- 


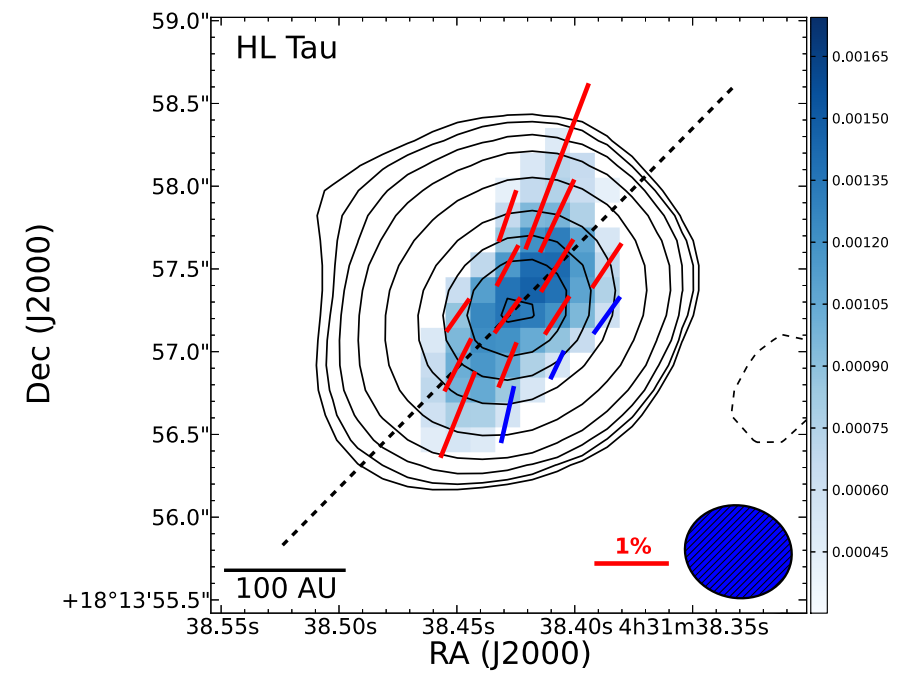

Figure 1: 1. Detected magnetic field morphology of HL Tau at $0.6^{\prime \prime}$ resolution. The polarization vectors have been rotated by $90^{\circ}$ to show the inferred magnetic field orientation. Red vectors are detections $>3 \sigma_{P}$ while blue vectors are detections between $2 \sigma_{P}$ and $3 \sigma_{P}$. We also do not show vectors when the signal-to-noise for Stokes $I$ is below 2. The sizes of the vector are proportional to the fractional polarization, $P$. Stokes $I$ contours are shown for $[-3,3,4,6,10,20,40,60,80$, 100] $\times \sigma_{I}$, where $\sigma_{I}=2.1 \mathrm{mJy}_{\text {beam }}{ }^{-}$. Color scale shows the polarized intensity with the color bar in $\mathrm{Jy}_{\text {beam }}{ }^{-}$. The dashed line shows the major axis of PA $=136^{\mathrm{c}} \underline{12}$. The synthesized beam is shown on the bottom right and has a size of $0.65^{\prime \prime} \times 0.56^{\prime \prime}$ and a PA $=79.5^{\circ}$. 


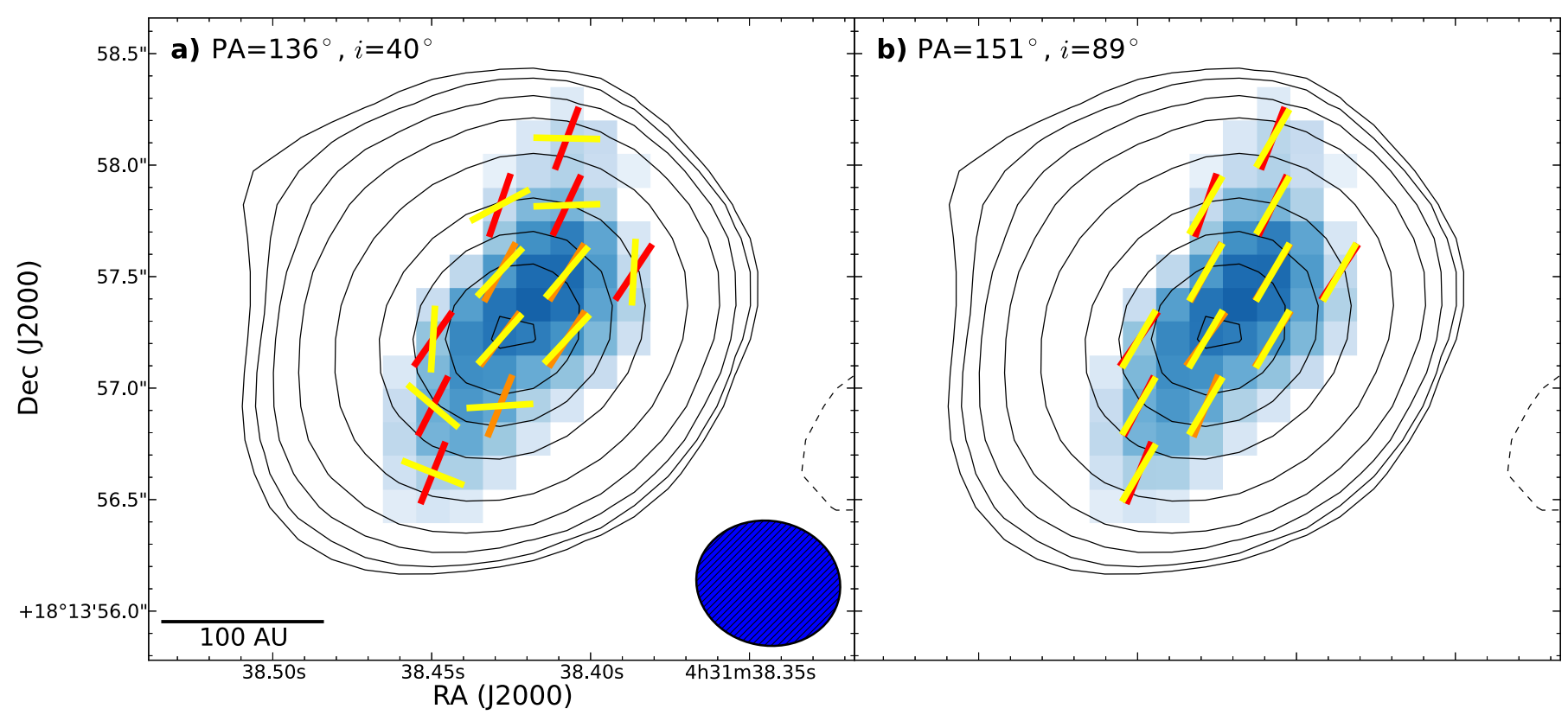

Figure 2: 2. Observed magnetic field morphology compared with models. The observed $3 \sigma$ and $5 \sigma$ detections are shown in red and orange, respectively, while modeled vectors are shown in yellow. Black contours, color scale, and beam size are the same as Figure 1. a) 100\% toroidal field model with $\mathrm{PA}=136^{\circ}$ and $i=40^{\circ}$. b) $100 \%$ toroidal field model with $\mathrm{PA}=151^{\circ}$ and $i=89^{\circ}$. 
ization in $0510+180$ was not very large, the consistency of polarization measurements of $0510+180$ between tracks made us confident that our calibration is accurate. For B-array tracks, we also saw that the bandpass calibrator, 3C454.3, showed consistency for polarization measurements for all the tracks. Other calibrators observed did not have polarization detected, signifying that our polarization detection of HL Tau is not a spurious detection. We also note that there was a slight difference in the polarization angle between the lower and upper sideband; this difference may be due to Faraday rotation and was almost constant for all tracks.

For bandpass calibration, C-array observations used 3C84 and B-array observations used 3C454.3. The phase calibrator used for both arrays was $0510+180$. Observations of MWC349, with an adopted flux of $2.1 \mathrm{Jy}^{30}$ provided the absolute scale for the flux calibration at $237 \mathrm{GHz}$ in most of the tracks. The bootstrapped flux of 0510+180 using MWC349 was consistent within 10\% and 15\% to bootstrapped fluxes using Mars and Uranus respectively in other tracks. Since planets are resolved at these resolutions, MWC349 is likely to have a more accurate flux calibration and was bootstrapped for all tracks. The absolute flux uncertainty is estimated to be $15 \%$, but only statistical uncertainties are discussed in this work. When imaging, natural weighting was used to maximize the sensitivity.

Detections of polarization were found in every HL Tau track with consistent polarization angles and measurements. Since polarization is calculated from Stokes $Q$ and $U$ and can only have positive values, there exists a bias in the polarized intensity; hence, all our polarization measurements have been de-biased 25 . The sensitivity in Stokes $I$ is limited by dynamic range rather than the flux sensitivity of the observations. The uncertainty in the absolute position angle of CARMA 
is approximately $3^{\sqrt{25}}$.

These high resolution interferometric observations are insensitive to large scale structure. Single dish and interferometric HL Tau observations find very similar compact fluxes, suggesting that the envelope dust continuum is negligible ${ }^{21}$, and the flux appears to come entirely from the $\operatorname{disk}^{12}$.

Also reported in this study are unpublished polarization observations (PA and $P$ ) of HL Tau from the SMA. These observations were taken in the compact configuration in October 2005 in the $345 \mathrm{GHz}$ atmospheric window with the Local Oscillator tuned to $341.5 \mathrm{GHz}$. The polarization data reduction process was done in the typical manner employed by the $\mathrm{SMA}^{31}$.

Linear Polarization Modeling. We employed a flared viscous accretion disk model that was constrained by high angular resolution data and a broad spectral energy distribution, detailed in another pape ${ }^{\sqrt{12}}$. The accretion disk model has a power-law density distribution with an exponential tapering, and the vertical density distribution is assumed as 1.5 times thicker than the hydrostatic equilibrium case. The temperature distributions are calculated by interpolation of two power-law distributions at the cold mid-plane, comparable to the results of the Monte-Carlo radiative code RADMC-3D 32 and at the surface based on radiation equilibrium: $T_{m}[K]=190(r / \mathrm{AU})^{-1} \quad$ and $T_{s}[K]=600(r / \mathrm{AU})^{-1}$. The disk parameters employed were the volume density power-law index $p=1.064$, the dust opacity spectral index $\beta=0.729$, the disk mass $M_{d}=0.1349 M_{\odot}$, the inner radius $R_{\text {in }}=2.4 \mathrm{AU}$, and the characteristic radius $R_{c}=78.9 \mathrm{AU}$. As described in the main text, we investigated various cases of different inclination and position angles. 
Our polarization modeling consists of toroidal and vertical magnetic fields. Instead of constraining the detailed morphology of magnetic fields, we intended to constrain which morphology is preferred. In order to achieve this goal, we examined 101 cases spanning over relative polarization fractions of the two orthogonal fields in steps of $1 \%$ (i.e., $100 \%$ toroidal, $99 \%$ toroidal and $1 \%$ vertical, $98 \%$ toroidal and $2 \%$ vertical, ...). For constructing linear polarization information, we built Stokes $I, Q$, and $U$ maps by numerically solving radiative transfer (necessary for a thick disk). In individual integral elements of radiative transfer along line of sight, we compute the intensity for $Q$ and $U$. The fractional intensities added up to the $Q$ and $U$ maps by an integral element are:

$$
\begin{gathered}
\Delta Q=\Delta I f_{p}\left(f_{\text {tor }} q_{\text {tor }}+f_{v e r} q_{v e r}\right) \\
\Delta U=\Delta I f_{p}\left(f_{\text {tor }} u_{t o r}+f_{v e r} u_{v e r}\right)
\end{gathered}
$$

where $f_{p}$ is a total polarization fraction $(\sqrt{Q+U} / I), f_{\text {tor }}$ and $f_{v e r}$ are relative fractions of the toroidal and vertical fields (e.g., $f_{\text {tor }}=0.7$ and $f_{\text {ver }}=0.3$ for $70 \%$ toroidal and $30 \%$ vertical fields), and $q_{t o r}$ and $u_{\text {tor }}$ are $\cos \left(2 \chi_{\text {tor }}\right)$ and $\sin \left(2 \chi_{\text {tor }}\right)$ respectively. $\chi$ is the angle of the magnetic field measured counterclockwise from the north. Similarly, $q_{v e r}$ and $u_{v e r}$ for vertical fields are $\cos \left(2 \chi_{v e r}\right)$ and $\sin \left(2 \chi_{v e r}\right)$. Since the disk is optically thin and we only care about the morphology, $f_{p}$ can be given an arbitrary value (e.g., 0.01 or 0.1 ). Note that the toroidal and vertical magnetic field vectors at each integral element have been tilted and rotated based on the inclination and the position angle of the model disk, before the calculation of the fractions. $Q$ and $U$ maps are convolved with the synthesized beam from the polarization observations, and the modeled position angles of the magnetic field morphology is created using $\chi=0.5 \tan ^{-}(U / Q)$. 
28. Sault, R. J., Teuben, P. J. \& Wright, M. C. H. A Retrospective View of MIRIAD. In Shaw, R. A., Payne, H. E. \& Hayes, J. J. E. (eds.) Astronomical Data Analysis Software and Systems IV, vol. 77 of Astronomical Society of the Pacific Conference Series, 433 (1995).

29. Witzel, A., Heeschen, D. S., Schalinski, C. \& Krichbaum, T. Kurzzeit-Variabilität extragalaktischer Radioquellen. Mitteilungen der Astronomischen Gesellschaft Hamburg 65, 239 (1986).

30. Tafoya, D., Gómez, Y. \& Rodríguez, L. F. Imaging MWC 349 from 7 Millimeters to 90 Centimeters. Astrophys. J. 610, 827834 (2004).

31. Marrone, D. P. \& Rao, R. The submillimeter array polarimeter. vol. 7020 of Society of PhotoOptical Instrumentation Engineers (SPIE) Conference Series (2008).

32. Dullemond, C. P. \& Dominik, C. Flaring vs. self-shadowed disks: The SEDs of Herbig Ae/Be stars. Astron. Astrophys. 417, 159168 (2004). 